\title{
Risk Factors for Contracting Malaria in Nvanga District, Zimbabwe: a Case Control Study
}

\author{
Article by Mashizha $\mathrm{S}^{1}$, Gombe $\mathrm{N} \mathrm{T}^{2}$, Gunda $\mathrm{T}^{3}$ \\ ${ }^{2}$ Ph.D. in Public Health, Texila America University, International Organisation for \\ Migration, Zimbabwe \\ E-mail:2ntgombe@Gmail.com
}

\begin{abstract}
Introduction: A review of Nyanga District malaria weekly surveillance data showed that malaria cases had surpassed the thresholds from week 4 confirming that an outbreak was underway. We investigated the outbreak to determine risk factors associated with contracting malaria.

Methods: A 1:1 Unmatched Case control study was carried out in Nyanga district. Interviewer administered questionnaires and WHO adapted Integrated Disease Surveillance and Response (IDSR) checklists were used to collect information on risk factors and on outbreak response. Data were analysed using Epi-Info 7 statistical software.

Results: Eighty three cases and 83 controls were enrolled into the study. Most (76\%) of the under 5 year cases came from villages across the border in Mozambique. Presence of stagnant water bodies near home [OR=11.06; 95\%CI $(2.32 ; 52.68)]$ was associated with contracting malaria. Consistently sleeping under a net [OR=0.13; 95\% CI (0.03; 0.59)] Use of mosquito repellents [OR $=0.16 ; 95 \%$ CI $(0.04 ; 0.58)]$ and having received health education on malaria in the past year $[O R=0.19 ; 95 \%$ CI $(0.07 ; 0.52)]$ were protective against contracting malaria.

Conclusion: The district was not prepared for the outbreak and the outbreak mainly affected children under five years from Mozambique. The risk factor for contracting malaria during the outbreak was presence of stagnant water bodies near homes which facilitated breeding of the vector. Consistently sleeping under a mosquito net, use of mosquito repellents and reported history of receiving health education on malaria in the past year were protective. As a result of this study health education was given to the community.
\end{abstract}

Keywords: Thresholds, Malaria, Case Control, Risk Factors

\section{Introduction}

Malaria is a disease caused by infection of the red blood cells with the protozoan parasites Plasmodium. There are four Plasmodium species that infect humans: P. falciparum, P. vivax, $P$. ovale and $P$. malariae. $P$. falciparum is the most virulent of the plasmodia and is responsible for majority of infections in Africa. The disease is transmitted through a bite from an infected female anopheles mosquito ${ }^{1}$.

Malaria can present as uncomplicated or complicated malaria. Uncomplicated malaria usually presents with fever, chills, general body weakness, headache and joint pains and at times abdominal pains and diarrhoea. Complicated malaria presents with any signs and symptoms of uncomplicated disease plus any of the danger signs which includes convulsions, coma, severe pallor, jaundice, inability to drink and passing dark or little urine. Complicated malaria is an emergency and is associated with high morbidity and mortality ${ }^{2}$. Malaria diagnosis is made through malaria parasite slide (MPs) and the Rapid Diagnostic Test (RDTs). In Zimbabwe, uncomplicated malaria is treated with Coartemether, Quinine and Doxycycline or Clindamycin while complicated with parenteral Quinine. ${ }^{2}$

Malaria is both preventable and curable, and mosquito bites can be averted. The disease can be eliminated, but resurgence is always a possible threat. Malaria control intervention 
Texila International Journal of Public Health

Volume 4, Issue 4, Dec 2016

strategies are case management, vector control, surveillance and research, social mobilization and advocacy and emergency preparedness and response.

Vector control methods used are use of insecticide treated nets (ITN), indoor residual spraying (IRS), environmental management and larviciding ${ }^{3}$.

Globally, 300-500 million malaria cases are recorded annually, and 90\% of these occur in Sub Saharan Africa. Between 1.1-2.7 million people die annually from malaria, and more than one million are children under five years. ${ }^{4}$

In 2010, the World Health Organisation (WHO) estimates that there were 3.3 billion people at risk of malaria and 216 million cases were reported; of these $81 \%$ were in Sub Saharan Africa ${ }^{5}$. Of the estimated 655,000 people who died of malaria, 91\% were in SubSaharan Africa and 86\% children under 5 years ${ }^{5}$.

Most people at risk of malaria in Africa live in areas where transmission is intense and continuous. They develop some degree of immunity to the disease with age and children under 5 years and pregnant women are most at risk ${ }^{4}$. A smaller proportion of people live in areas of seasonal and unstable transmission. They have lower levels of immunity and hence all age groups are vulnerable to seasonal transmission and epidemics ${ }^{4}$.

In Zimbabwe malaria is a public health concern. More than half of the population resides in malaria transmission risk areas. Out of the 56 districts, malaria transmission occurs in $42^{6}$. This stratification was done in 2002 based on the national parasite prevalence survey, health management information system data, entomological data and expert opinion. ${ }^{6}$

Zimbabwe is divided into three strata. Free malaria transmission areas, where there is no local transmission, unstable malaria transmission areas were transmission is seasonal and low. These areas are prone to epidemics because the population does not have some degree of immunity to malaria and all age groups are at risk. Stable transmission areas are highly endemic and the local people develop some degree of immunity. Children under 5 years, pregnant women are most affected and are at risk of developing complicated malaria. ${ }^{6}$

Nyanga is one of the seven districts in Manical and Province. It borders with Mutoko District to the north, Mutasa District to the south and Mozambique to the east. It is characterised by moderate transmission of malaria which is seasonal. Most of the malaria cases are seen at Rural Health Centres (RHC) centres. Complicated cases are referred to the three hospitals in the district.

The malaria control measures being implemented in Nyanga include vector control through indoor residual spraying (IRS) and distribution of long lasting insecticide treated nets (LLITNs), intermittent preventive therapy (IPT) in pregnant women and case management. The district also distributes anti-malaria drugs and malaria tests kits (RDT) to community based health workers who include Village Health Workers (VHW) and ACT Holders. In addition, there is continuing health education to the community about malaria related issues.

Chatindo clinic is situated $80 \mathrm{~km}$ from Nyanga district hospital. The estimated clinic catchment population is 5750. It is situated in ward 11. The clinic's attachment area includes wards 11 and part of ward 10. The clinic is situated within a walking distance from the international border with Mozambique, so people who live along the border on the Mozambican side use this health facility. The referral center for the clinic is Regina Coeli Mission Hospital.

A review of the Nyanga District Malaria Weekly Disease Surveillance System (WDSS) data revealed that malaria cases at Chatindo clinic had surpassed the thresholds from week 4. For weeks four to six the clinic recorded 88; 114 and 108 cases of malaria against thresholds of 81; 52 and 96 respectively (Figure 1). The district Rapid Response Team went to investigate on the $2^{\text {nd }}$ of February 2013 and confirmed an outbreak. We investigated the outbreak to determine risk factors associated with contracting the disease. 
Texila International Journal of Public Health

Volume 4, Issue 4, Dec 2016

\section{Materials and Methods}

An unmatched 1:1case-control study was carried out at Chatindo Rural Health Centre and its catchment area among residents of Chatindo ward and those residing in the clinic's catchment area. A case was defined as a person residing in Chatindo or the clinic's catchment area, who presented to Chatindo clinic with sudden onset of intermittent fever with shivering, sweating, headache, joint and muscle pains, nausea and vomiting, chills and body weakness and would have been diagnosed of malaria using rapid diagnostic test (RDT) during the period 20 January 2013 to 24 February 2013 and a control as a person residing in Chatindo or the clinic's catchment area who was attended at the clinic during the period 20 January 2013 to 24 February 2013 and tested negative for Malaria using malaria rapid diagnostic test.

A sample size of 166 respondents comprising 83 cases and 83 controls was calculated using Epi Info 7 with the following assumptions, 36.4\% exposure in controls and 59.15\% exposure in cases that stay in a house that was not sprayed, Odds Ratio of 2.53 (Maenzanise et al 2004), 95\% confidence and $80 \%$ power.

Cases were selected from the clinic register. A list containing all malaria cases seen at Chatindo clinic from 20 January 2013 to 24 February 2013 was prepared and used as a sampling frame. Individual cases were selected randomly from the sampling frame using random number tables until 83 cases were selected. Controls were also selected from the same register. A list of people who were attended at the clinic from 20 January 2013 to 24 February 2013 who did not develop malaria and tested negative for malaria using RDT was prepared and used as a sampling frame. Individual controls were selected randomly using random number tables until 83 controls were selected. Controls that were selected from the T12 register that came from Mozambique were called to the clinic for interviews using village health workers and those that resided in Zimbabwe were followed to their homes.

Permission to conduct the study was sought from the Provincial Medical Director (PMD) Manicaland Province, the District Medical Officer Nyanga and the Health Studies Office. Informed written consent was obtained from all participants. Participants' right to participate was respected. All information was kept confidential and completed questionnaires were kept under lock and key and permission to take pictures was sought and obtained from individual participants.

Interviewer administered questionnaires were used to collect data on demographic characteristics, risk factors, details of illness and treatment given. The questionnaire was also used to assess knowledge, attitudes and practises of cases and controls towards malaria prevention. Data on practices which included personal protection methods, use of mosquito repellents, presence, condition and use of insecticide treated mosquito nets were also collected. Medical records were reviewed to verify data on the treatments given and an Integrated Disease Surveillance and Response (IDSR) adapted check list was used to assess preparedness of district health team in malaria outbreak.

Pre testing of the study was done one week before carrying out the study at Nyamaropa clinic which is situated $10 \mathrm{~km}$ from Chatindo clinic. Data collection tools were assessed for the quality of answers to the questions, the time needed to administer the questionnaire, the willingness of respondents to answer questions and appropriateness of the answers and adjustments were made accordingly.

Epi Info statistical software was used to analyse data. We performed chi-square test for association; calculate odds ratios and their confidence intervals as well as p-values. Stratified analysis was carried out to check and control for confounders and assess for effect modification. Multivariate analysis (logistic) regression was used to identify independent risk factors for contracting malaria and to control for confounding variables

\section{Results}

Eighty three cases and 83 controls were enrolled into the study. The outbreak started in week in week 4 of 2013 when the clinic recorded 108 malaria cases against a threshold of 88 
Texila International Journal of Public Health

Volume 4, Issue 4, Dec 2016

and was only detected in week 5 by the District Health Information Officer when he calculated the thresholds for the Clinic. It was declared over in week 10 (figure1).

Socio- demographic characteristics of Cases and Controls were comparable, there was no statistical significant difference between cases and controls on variables age group, sex, Occupation, place of residence and level of education (Table 1).

Knowledge of controls on malaria was better than of cases. There were statistical significant differences on knowledge among cases and controls on malaria prevention practices.

Socio demographic characteristics associated with contracting Malaria in Chatindo were age less than five years OR 3.24 (0.84-12.44), place of residence in Mozambique OR $1.27(0.58-2.78)$ and male sex OR $1.37(0.72-2.59$ though not statistically significant however reported history of receiving health education on Malaria in the past year OR 0.15(0.07-0.30) was significantly protective against contracting Malaria

Personal and environmental factors associated with contracting malaria in Chatindo were potential stagnant water body around home OR 5.9(2.50-13.80), presence of tall grass and bushes around home OR 2.63(0.89-7.86), irrigating during the evening OR 2.12(1.07-4.21), sitting outside the house at night OR 2.20(1.15-4.20) and wearing short sleeved clothes during the night OR 5.7(2.25-13.47) were significantly associated with contracting Malaria. However reported sleeping under a mosquito net OR 0.31(0.16-0.63), reported consistently sleeping under a mosquito net OR 0.16(0.04-0.56), staying in a house that was sprayed in the last IRS season OR $0.46(0.21-0.97)$ and use of mosquito repellents $0.17(0.07-0.40)$ was protective

On logistic regression, presence of stagnant water bodies near the homestead OR 11.06(2.32-52.68), consistently sleeping under a mosquito net OR $0.13(0.03-0.59)$, use of mosquito repellents OR 0.16(0.04-0.58) and having received health education in the past year OR 0.19(0.07-0.52) were factors independently associated with contracting malaria in this study (Table 2).

The common presentation signs and symptoms were headache (73.5\%) followed by fever (49.4\%) general body weakness (45.8\%), shivering (25.3\%), muscle and joint paints (22.9\%) and nausea and vomiting (13.3\%) the least was diarrhea(1.2\%) (Figure 2). All cases were diagnosed using RDT and were managed on Coartemether as per national malaria treatment guidelines. There were no reported complications or fatalities among cases interviewed.

Regarding Malaria outbreak preparedness, health workers had not been trained in IDSR, there were no IDSR guidelines at the clinic, malaria thresholds were not in place, and EPR plans were not in place before, during and after the outbreak. Malaria thresholds were not in place they were only calculated at the district and send to the clinic on 30 January 2013. The IRS coverage (76\%) was below the program target of $85 \%$.

\section{Discussion}

In this study majority of the cases came were imported. Children under five years are at increased risk of contracting malaria because their immune system is still developing ${ }^{4}$. So in a community like Mozambican villages were malaria vector control interventions (IRS and Mosquito net distribution) are non-existent, children under five years are at more risk of contracting malaria than the general population. This could explain the high proportion of cases in the less than five years age group coming from Mozambique. Malaria vector control interventions like IRS and Net distribution which were done in Chatindo in November 2012 and August 2010 respectively were not done on the Mozambican side despite the close proximity of these communities.

Mosquito net usage and staying in a house that was sprayed in the last IRS season were significantly protective against contracting malaria. This could explain the high proportion of cases from Mozambique. These finding are consistent with findings by Mpofu et al in 2006 identified consistently sleeping under a net and staying in a house that was sprayed (IRS) as significantly protective against contracting malaria. ${ }^{8}$ 
From the villages in Zimbabwe, the majority of the cases came from the Irrigation areas A and $\mathrm{B}$, and it mainly affected the economic active age group 15 years and above. This age group is involved in the farming activities in the irrigation area and in some instances people knock off from the fields after dawn and irrigate during the night. Evening outdoor activities like irrigating in the evening or during the night were found in the study as a significant risk factor for contracting malaria in Chatindo. This could explain the higher proportion of cases in the age group 15 and above contracting malaria in the irrigation areas. Dhege and others in 2012 also found irrigation/watering at night as a significant risk factor for contracting malaria $^{10}$.

Controls were more knowledgeable on malaria prevention practices than cases. Having received health education on malaria in the past year was protective against contracting malaria in this study. Health education can result in malaria prevention practices like consistent use of a net and uptake of IRS which have been found to be protective in this study and eliminating potential breeding sites by filling of stagnant water bodies and potholes near home and cutting tall grass and bushes around home which were significant risk factor for contracting malaria in this study. Provision of health education on malaria can result in increased awareness on malaria prevention practices, of which when done will result in reduced risk of contracting the disease.

Presence of stagnant water bodies near home was significantly associated with contracting malaria in the study. This is similar to finding by Marape and others in 2008 who also found stagnant water bodies near home as risk factors for contracting malaria. ${ }^{7}$

Rains also promote the growth of bushes and grass around homes which also provides resting places for adult mosquitoes as well as breeding places. Thick vegetation, tall grass and bushes around the home were also identified as a risk factor for contracting malaria in Chatindo. This is consistent with findings by Chikwanha and others in 2010 in Mazowe malaria outbreak investigation that identified presence of tall grass and thick vegetation around home as a significant risk factor for contracting malaria. ${ }^{9}$

Under 5 year age group was associated with contracting Malaria, so malaria prevention interventions like mosquito net distribution should target under five years old children.

Chatindo clinic was not prepared for the malaria outbreak. Heath workers were not trained in IDSR, there were no IDSR guidelines at the clinic, malaria thresholds were not in place, and Epidemic Preparedness and Response (EPR) plans were not in place before, during, and after the outbreak. Malaria thresholds were not in place they were only calculated at the district and send to the clinic on 30 January 2013 after the outbreak had started. This shows lack of supervision by the DHE which is supposed to make sure that EPR plans and epidemic thresholds are in place at all clinics. This will prevent late detection of the outbreaks which can result in morbidity and mortality that could be prevented by early detection and prompt appropriate interventions.

Although IRS was carried out in Chatindo, the coverage was low (76\%) for public health impact, for a public health intervention to achieve desirable outcome the coverage should be at least above $85 \%$. This was due to refusals and locked rooms. Some of the reasons were that people were busy in the fields and the chemical was not effective because it was not killing the cockroaches.

There are no structures for inter district collaboration between Nyanga district and Mozambique, This makes it difficult to coordinate vector control interventions between Nyanga district and Mozambique. In order to harmonize malaria control interventions structures for collaboration should be put in place first.

\section{Conclusion}

Chatindo Clinic was not prepared for the outbreak and the risk factor for contracting malaria during the outbreak in Chatindo was presence of stagnant water bodies near homes. However, consistently sleeping under a mosquito net, use of mosquito repellents and reported history of receiving health education on malaria in the past year were protective. 
Texila International Journal of Public Health

Volume 4, Issue 4, Dec 2016

\section{Recommendations}

In view of the findings, sstructures for collaboration between Nyanga District and Mozambique should be put in place to enable harmonisation of malaria vector control intervention such as IRS and ITNs in the two districts. All clinics should have malaria epidemic thresholds in place to improve outbreak preparedness and the District Medical Officer should arrange for IDSR trainings and ensure that emergency preparedness and response plans are in place at all clinics so as to detect outbreaks early.

\section{References}

[1] Chikwanha TM, Chinhengo TP, Hanyana N : Malaria Outbreak Investigation Mazowe District Mashonaland Central Province, Zimbabwe 2010 (Unpublished)

[2] Centre for Disease Control and Prevention. Malaria accessed from www.cdc.gov/malaria/about/facts.html on 14/02/13

[3] Dhege C, Zizhou S T, Muyengwa P. Malaria Outbreak investigation in Mudzi District 2012.(unpublished)

[4] Ministry of Health and Child Welfare, Zimbabwe: Preliminary Malaria Stratification Report, 2002 accessed from www.rbm.who.int/countryaction/.../naZimbabwe.pdf on 14/02/13

[5] Marape G, Mpeta ET, Ndlovu N: Factors associated with contracting malaria at Mbembeswana, 10 March to 25 April 2008, Zimbabwe (Unpublished)

[6] Mpofu M, Nkomo B.M.M, Kulkarni R, Mabaera B: Malaria Outbreak in Tsholotsho District in Matabeleland North Province, Zimbabwe, 2006 (Unpublished)

[7] Ministry of Health and Child Welfare Zimbabwe: World Health Organisation, United Nations Development Programme and the Global Fund. Guidelines for Management of Malaria in Zimbabwe, Revised December 2009.

[8] Ministry of Health and Child Welfare, World Health Organisation, united nations Development Programme and the Global Fund. Guidelines for management of malaria in Zimbabwe, revised December 2009.

[9] Maenzanise S R, Kusangaya O, Chirenda J, Tshimanga M : Malaria Outbreak Investigation In Msampakaruma, Kariba District, 2004 (Unpublished)

[10] Rasheed S. et al (2000), Economic impact of febrile morbidity and use of permethrinimpregnated bed nets in a malarious area II, Determinants of febrile illness and the cost of their treatment and malaria prevention,. AMJ Trop. Med Hyg. 62: 181-6

[11] WHO: Guidelines for treatment of malaria, Second edition, WHO Library Cataloguing in Publication Data, 2010.

[12] WHO: A commitment to supporting national programs for greater coverage of Malaria control interventions, Annual Report 2006, World Health Organization, 2006

[13] WHO: Malaria Case Management Operational Manual, World Health Organization, Geneva, 2009

[14] World Health Organization :World Malaria Report, 2011, Geneva, 2011

[15] World Health Organization, Global Malaria Action Plan, Roll Back Malaria Partnership, World Health Organization, Geneva, 2008 accessed from http://rbm.who.int/gmap/2-3.html on 14/01/13 
Texila International Journal of Public Health

Volume 4, Issue 4, Dec 2016

\section{Tables and figures}

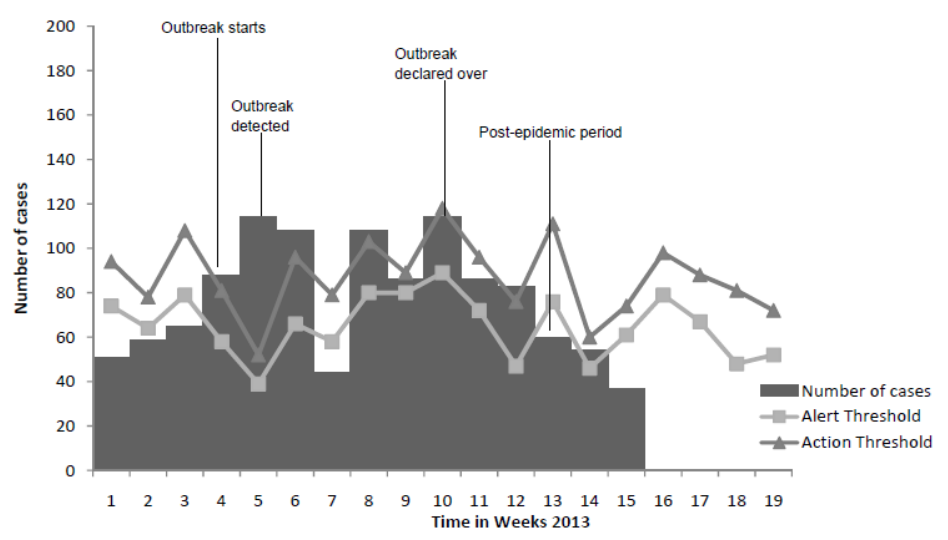

Figure 1:Distribution of Malaria Cases against the Thresholds in Chatindo 2013

Table 1: Demographic Characteristics of Cases and Controls in Chatindo Malaria Outbreak Investigation in 2013

\begin{tabular}{|c|c|c|c|c|}
\hline Variable & Category & $\begin{array}{c}\text { Cases } \\
n=83(\%) \\
\end{array}$ & $\begin{array}{c}\text { Controls } \\
\mathrm{n}=83(\%) \\
\end{array}$ & P-value \\
\hline \multirow[t]{2}{*}{ Sex } & Male & $32(39.8)$ & $27(32.5)$ & 0.33 \\
\hline & Female & $50(60.2)$ & $54(67.5)$ & \\
\hline \multirow[t]{3}{*}{ Educational level } & None & $15(18.1)$ & 11(13.3) & 0.51 \\
\hline & Primary & $36(43.4)$ & $43(51.8)$ & \\
\hline & Secondary & $32(38.5)$ & $29(34.9)$ & \\
\hline \multirow[t]{4}{*}{ Religion } & Apostolic & $24(28.9)$ & $32(38.6)$ & 0.02 \\
\hline & Pentecostal & 31(37.3) & $22(26.5)$ & \\
\hline & Orthodox & $14(16.9)$ & $24(28.9)$ & \\
\hline & None & 14(16.9) & $5(6.0)$ & \\
\hline \multirow{2}{*}{ Occupation } & Formal & $7(8.4)$ & $9(10.8)$ & 0.50 \\
\hline & Informal & $76(91.6)$ & $74(89.2)$ & \\
\hline \multirow[t]{5}{*}{ Age group } & $<$ 5years & $9(10.8)$ & $3(3.6)$ & 0.13 \\
\hline & $5-14$ yrs & $27(32.5)$ & $19(22.9)$ & \\
\hline & $15-24 y r s$ & $17(20.5)$ & $17(20.5)$ & \\
\hline & $25-49$ yrs & $21(25.3)$ & $32(38.6)$ & \\
\hline & $50+$ yrs & $9(10.8)$ & $12(14.5)$ & \\
\hline \multirow[t]{4}{*}{ Place of Residence } & Irrigation A & 24(28.9) & 33(39.80 & 0.06 \\
\hline & Irrigation B & $25(30.1)$ & $30(36.10$ & \\
\hline & Mozambique & $17(20.5)$ & $6(7.2)$ & \\
\hline & Nyadowa & $17(20.5)$ & $14(16.9)$ & \\
\hline
\end{tabular}

Table 2: Factors independently associated with Contracting Malaria in Chatindo, 2013

\begin{tabular}{llll}
\hline \multicolumn{1}{c}{ Factor } & $\begin{array}{c}\text { Crude Odds Ratio } \\
(\mathbf{9 5 \% C I )}\end{array}$ & $\begin{array}{c}\text { Adjusted Odds Ratio } \\
\text { (95\%CI) }\end{array}$ & P-value \\
\hline $\begin{array}{l}\text { Presence of stagnant water } \\
\text { bodies near homestead }\end{array}$ & $5.9(2.50-13.80)$ & $\mathbf{1 1 . 0 6}(2.32-52.68)$ & 0.003 \\
$\begin{array}{l}\text { Reported consistently sleeping } \\
\text { under a mosquito net }\end{array}$ & $0.16(0.04-0.56)$ & $\mathbf{0 . 1 3}(0.03-0.59)$ & 0.008 \\
$\begin{array}{l}\text { Use of mosquito repellents } \\
\begin{array}{l}\text { Receiving health education on } \\
\text { malaria in the past year }\end{array}\end{array}$ & $0.17(0.07-0.40)$ & $\mathbf{0 . 1 6}(0.04-0.58)$ & 0.005 \\
\hline
\end{tabular}


Texila International Journal of Public Health

Volume 4, Issue 4, Dec 2016

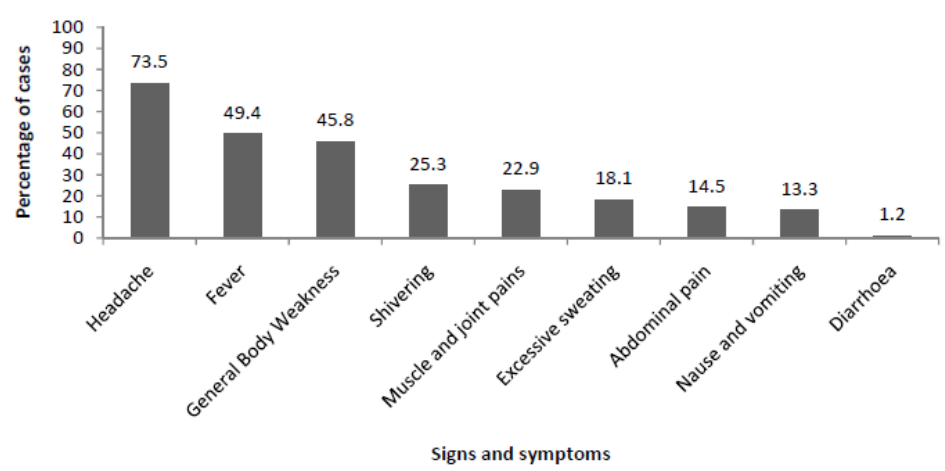

Figure 2: Presenting Signs and Symptoms for Malaria Cases in Chatindo 2013

Table 3: Treatment Given to Malaria Cases at Chatindo Clinic, Nyanga during the Outbreak in 2013

\begin{tabular}{lll}
\hline \multicolumn{1}{c}{ Treatment given } & $\mathbf{n = 8 3}(\mathbf{\% )}$ \\
\hline Coartemether & $83(100)$ & \\
Cases attended by nurses & $67(80.7)$ & \\
Cases attended by nurse aides & $16(19.3)$ & \\
Paracetamol & $69(83.1)$ \\
Intravenous quinine & 0 \\
Intravenous fluids & 0 \\
Complications & 0 & \\
Case fatality & 0 & \\
\hline
\end{tabular}

Table 4: Nyanga District Outbreak Response, 2013

\begin{tabular}{llll}
\hline \multicolumn{1}{c}{ Indicator } & Target & $\begin{array}{c}\text { Achieved } \\
\text { timeliness }\end{array}$ & \multicolumn{1}{c}{ Comments } \\
\hline $\begin{array}{l}\text { Outbreak identification and } \\
\text { reporting to district level }\end{array}$ & within 24hrs & Nil & $\begin{array}{l}\text { Outbreak was detected by } \\
\text { the DHE 48hrs later } \\
\text { (Delayed) }\end{array}$ \\
$\begin{array}{l}\text { District notification and field } \\
\text { investigation }\end{array}$ & within 48hrs & $72 \mathrm{hrs}$ & $\begin{array}{l}\text { DHE visited healthcare } \\
\text { centres to verify outbreak } \\
\text { (delayed) } \\
\text { Poor response }\end{array}$ \\
$\begin{array}{l}\text { District level concrete } \\
\text { response by district }\end{array}$ & within 48hrs & 96 hours & Achieved \\
$\begin{array}{l}\text { Start of outbreak and } \\
\text { submission of outbreak } \\
\text { report to higher levels }\end{array}$ & within 2weeks & 10 days & \\
\hline
\end{tabular}

